\title{
Analysis of the charmed mesons $D_{1}^{*}(2680), D_{3}^{*}(2760)$ and $D_{2}^{*}(3000)$
}

\author{
Guo-Liang $\mathrm{Yu}^{1} *$ Zhi-Gang Wang ${ }^{1} \doteq$ and ZhenYu $\mathrm{Li}^{2}$ \\ ${ }^{1}$ Department of Mathematics and Physics, North China Electric power university, \\ Baoding 071003, People's Republic of China \\ 2 School of Physics and Electronic Science, Guizhou Normal College, \\ Guiyang 550018, People's Republic of China
}

(Dated: May 26, 2022)

\begin{abstract}
In this work, we systematically study the strong decay behavior of the charmed mesons $D_{1}^{*}(2680)$, $D_{3}^{*}(2760)$ and $D_{2}^{*}(3000)$ reported by the LHCb collaboration. By comparing the masses and the decay properties with the results of the experiment, we assign these newly observed mesons as the $2 S \frac{1}{2} 1^{-}, 1 D \frac{5}{2} 3^{-}$and $1 F \frac{5}{2} 2^{+}$states respectively. As a byproduct, we also study the strong decays of the unobserved $2 P \frac{3}{2} 2^{+}, 2 F \frac{5}{2} 2^{+}$and $3 P \frac{3}{2} 2^{+}$charmed mesons, which is useful for future experiments in searching for these charmed mesons.

PACS numbers: 13.25.Ft; 14.40.Lb
\end{abstract}

\section{Introduction}

Recently, the LHCb Collaboration studied the resonant substructures of $B^{-} \rightarrow D^{+} \pi^{-} \pi^{-}$decays in a data sample corresponding to $3.0 \mathrm{fb}^{-1}$ of pp collision data recorded by the LHCb experiment during 2011 and 2012. By a Dalitz plot analysis technique, the presence of resonances with spins 1, 2 and 3 at the $D^{+} \pi^{-}$mass spectrum were confirmed [1]. Their analysis indicated that these resonances are mainly from the contributions of $D_{2}^{*}(2460), D_{1}^{*}(2680), D_{3}^{*}(2760)$ and $D_{2}^{*}(3000)$ charmed mesons. The masses and decay widths of these mesons are

$$
\begin{aligned}
& D_{2}^{*}(2460): M=2463.7 \pm 0.4 \pm 0.4 \pm 0.6 \mathrm{MeV}, \Gamma=47.0 \pm 0.8 \pm 0.9 \pm 0.3 \mathrm{MeV} \\
& D_{1}^{*}(2680): M=2681.1 \pm 5.6 \pm 4.9 \pm 13.1 \mathrm{MeV}, \Gamma=186.7 \pm 8.5 \pm 8.6 \pm 8.2 \mathrm{MeV} \\
& D_{3}^{*}(2760): M=2775.5 \pm 4.5 \pm 4.5 \pm 4.7 \mathrm{MeV}, \Gamma=95.3 \pm 9.6 \pm 7.9 \pm 33.1 \mathrm{MeV} \\
& D_{2}^{*}(3000): M=3214 \pm 29 \pm 33 \pm 36 \mathrm{MeV}, \Gamma=186 \pm 38 \pm 34 \pm 63 \mathrm{MeV}
\end{aligned}
$$

Actually, people have found many other charmed mesons before these discoveries [2 9$]$, which have greatly enriched the charmonium spectra. On the other hand, these discoveries also shed more light on our knowledge about the essence of the elementary particles in the micro-world. For $D_{2}^{*}(2460)$ as an example, it has been well established previously and the $1 P \frac{3}{2} 2^{+}$assignment is strongly favored [10]. We studied the nature of the states $D_{1}^{*}(2680), D_{3}^{*}(2760)$ and $D_{2}^{*}(3000)$ in our previous work using

*Electronic address: yuguoliang2011@163.com

†Electronic address: zgwang@aliyun.com 
the heavy meson effective theory [1]]. Some of the strong decay behavior have also been studied in which the calculated ratios among the decay widths can be used to confirm or reject the assignments of the newly observed charmed mesons. The decay behavior of the $D_{2}^{*}(3000)$ charmed meson was also analyzed in reference [12], where it was assigned as the $2^{3} F_{2}$ or $3^{3} P_{2}$ states. In order to identify the $D_{1}^{*}(2680), D_{3}^{*}(2760)$ and $D_{2}^{*}(3000)$ and give more specific decay widths and the ratios, we further analyze the strong decay properties of these newly observed charmed mesons using the ${ }^{3} P_{0}$ decay model.

The ${ }^{3} P_{0}$ decay model is known as quark pair creation model (QPC) which was firstly introduced by Micu [13] in 1969. An important feature of the this decay model, apart from its simplicity, is that it provides the gross features of several transitions with two parameters, the pair-crestion strength $\gamma$ and the oscillator parameter $R$, which can be fitted to the experimental data. Soon after the introduction of the ${ }^{3} P_{0}$ model, it was further developed by other collaborations [14, 15]. This model, extensively applied to the decays of light mesons and baryons [16 25], has been applied to evaluate the strong decays of heavy meson in the charmonium [26 28], bottomonium [28, 29], and open-charm sectors [30, 31].

Just as what we have analyzed [11], the mesons of $D_{1}^{*}(2680), D^{*}(2600)$ and $D_{I}^{*}(2650)$ have the similar mass and width [32, 33], and can be assigned to be the same states $2 S \frac{1}{2} 1^{-}$[34 38 ]. Based on the same analysis, $D_{3}^{*}(2760)^{0}, D^{*}(2760)^{0}, D_{J}^{*}(2760)^{0}$ may be the same particle, and can be assigned to be the $1 D \frac{5}{2} 3^{-}$state $[32-40]$. As for $D_{2}^{*}(3000)$, it can be a $P$ wave and $F$ wave charmed meson. Its mass can be calculated by different theoretical models, such as the relativized quark model based on a universal one-gluon exchange plus linear confinement potential [41], the relativistic quark model includes the leading order $1 / M_{h}$ corrections [42], the QCD-motivated relativistic quark model based on the quasipotential approach [43]. According to these calculations, $1 F \frac{5}{2} 2^{+}, 2 P \frac{3}{2} 2^{+}, 2 F \frac{5}{2} 2^{+}$and $3 P \frac{3}{2} 2^{+}$can also be assigned as the candidates of the possible states of the charmed meson $D_{2}^{*}(3000)$.

To further verify the states of $D_{1}^{*}(2680)$ and $D_{3}^{*}(2760)$ and check the possibilities of different assignments of the $D_{2}^{*}(3000)$, we give a systematic analysis of the decay behaviors about these charmed mesons. The article is arranged as follows: In section 2 , the brief review of the ${ }^{3} P_{0}$ decay model is given (For the detailed review see Refs. 15, 17, 18, 20]); in Sec.3, we study the strong decays of the charmed mesons $D_{1}^{*}(2680), D_{3}^{*}(2760)$ and $D_{2}^{*}(3000)$ observed by the LHCb collaboration with the ${ }^{3} P_{0}$ decay model; in Sec.4, we present our conclusions.

\section{METHOD}

\subsection{The decay model}

The ${ }^{3} P_{0}$ decay model assumes that a quark-antiquark pair is created from the vacuum with the corresponding quantum number $0^{++}$. This new $q \bar{q}$ together with the $q \bar{q}$ within the initial meson regroups into two outgoing mesons in all possible arrangements for the meson decay process $A \rightarrow B C$ 


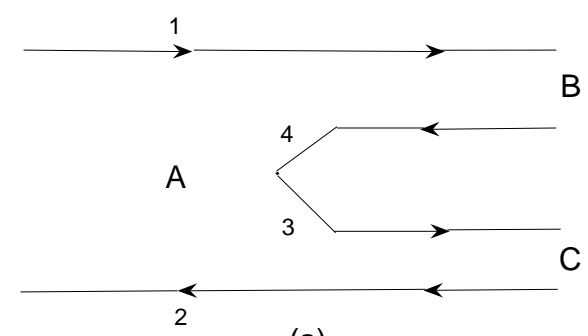

(a)

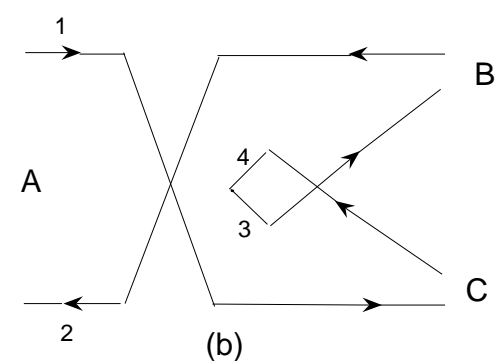

(b)

FIG. 1: The two possible decay processes of $A \rightarrow B C$ in the ${ }^{3} P_{0}$ model.

as shown in Fig. 1.

In the nonrelativistic limit, the transition operator of this process can be expressed as

$$
T=-3 \gamma \sum_{m}\langle 1 m 1-m \mid 00\rangle \int d^{3} \vec{p}_{3} d^{3} \vec{p}_{4} \delta^{3}\left(\vec{p}_{3}+\vec{p}_{4}\right) \mathcal{Y}_{1}^{m}\left(\frac{\vec{p}_{3}-\vec{p}_{4}}{2}\right) \chi_{1-m}^{34} \varphi_{0}^{34} \omega_{0}^{34} b_{3}^{\dagger}\left(\vec{p}_{3}\right) d_{4}^{\dagger}\left(\vec{p}_{4}\right)
$$

where the dimensionless parameter $\gamma$ denotes the creation strength of the quark-antiquark $q_{3} \bar{q}_{4}$ pair. $\vec{p}_{3}$ and $\vec{p}_{4}$ are the momenta of this quark-antiquark pair. Its flavor, color, and spin wave functions are represented by $\varphi_{0}^{34}, \omega_{0}^{34}$, and $\chi_{1-m}^{34}$, respectively. $\mathcal{Y}_{1}^{m}(\vec{p}) \equiv|\vec{p}|^{1} Y_{1}^{m}\left(\theta_{p}, \phi_{p}\right)$ is a solid harmonic polynomial corresponding to the p-wave quark pair.

In the center of mass frame of parent meson $A$, the helicity amplitude $\mathcal{M}^{M_{J_{A}} M_{J_{B}} M_{J_{C}}}$ of the decay process $A \rightarrow B C$ is written as

$$
\begin{aligned}
\mathcal{M}^{M_{J_{A}} M_{J_{B}} M_{J_{C}}(\vec{P})=} & \gamma \sqrt{8 E_{A} E_{B} E_{C}} \sum_{\substack{M_{L_{A}}, M_{S_{A}} \\
M_{L_{B}}, M_{S_{B}} \\
M_{L_{C}}, M_{S_{C}}, m}}\left\langle L_{A} M_{L_{A}} S_{A} M_{S_{A}} \mid J_{A} M_{J_{A}}\right\rangle\left\langle L_{B} M_{L_{B}} S_{B} M_{S_{B}} \mid J_{B} M_{J_{B}}\right\rangle \\
& \times\left\langle L_{C} M_{L_{C}} S_{C} M_{S_{C}} \mid J_{C} M_{J_{C}}\right\rangle\langle 1 m 1-m \mid 00\rangle\left\langle\chi_{S_{B} M_{S_{B}}}^{14} \chi_{S_{C} M_{S_{C}}}^{32} \mid \chi_{S_{A} M_{S_{A}}}^{12} \chi_{1-m}^{34}\right\rangle \\
& \times\left[\left\langle\phi_{B}^{14} \phi_{C}^{32} \mid \phi_{A}^{12} \phi_{0}^{34}\right\rangle I\left(\vec{P}, m_{1}, m_{2}, m_{3}\right)\right. \\
& \left.+(-1)^{1+S_{A}+S_{B}+S_{C}}\left\langle\phi_{B}^{32} \phi_{C}^{14} \mid \phi_{A}^{12} \phi_{0}^{34}\right\rangle I\left(-\vec{P}, m_{2}, m_{1}, m_{3}\right)\right]
\end{aligned}
$$

where the spatial integral is defined as

$$
\begin{aligned}
I\left(\vec{P}, m_{1}, m_{2}, m_{3}\right)= & \int d^{3} \vec{p} \psi_{n_{B} L_{B} M_{L_{B}}}^{*}\left(\frac{m_{3}}{m_{1}+m_{2}} \vec{P}_{B}+\vec{p}\right) \psi_{n_{C} L_{C} M_{L_{C}}}^{*}\left(\frac{m_{3}}{m_{2}+m_{3}} \vec{P}_{B}+\vec{p}\right) \\
& \times \psi_{n_{A} L_{A} M_{L_{A}}}\left(\vec{P}_{B}+\vec{p}\right) \mathcal{Y}_{1}^{m}(\vec{p})
\end{aligned}
$$


where $\vec{P}=\vec{P}_{B}=-\vec{P}_{C}, \vec{p}=\vec{p}_{3}, m_{3}$ is the mass of the created quark $q_{3}$, the simple harmonic oscillator (SHO) approximation is used for the meson space wave functions:

$$
\Psi_{n L M_{L}}(\vec{p})=(-1)^{n}(-i)^{L} R^{L+\frac{3}{2}} \sqrt{\frac{2 n !}{\Gamma\left(n+L+\frac{3}{2}\right)}} \exp \left(-\frac{R^{2} p^{2}}{2}\right) L_{n}^{L+\frac{1}{2}}\left(R^{2} p^{2}\right) \mathcal{Y}_{L M_{L}}(\vec{p})
$$

The partial wave amplitudes are related to the helicity amplitudes by [45]

$$
\mathcal{M}^{J L}(\vec{P})=\frac{\sqrt{4 \pi(2 L+1)}}{2 J_{A}+1} \sum_{M_{J_{B}} M_{J_{C}}}\left\langle L 0 J M_{J_{A}} \mid J_{A} M_{J_{A}}\right\rangle\left\langle J_{B} M_{J_{B}} J_{C} M_{J_{C}} \mid J M_{J_{A}}\right\rangle \mathcal{M}^{M_{J_{A}} M_{J_{B}} M_{J_{C}}(\vec{P})}
$$

where $M_{J_{A}}=M_{J_{B}}+M_{J_{C}}, \mathbf{J}_{\mathbf{A}}=\mathbf{J}_{\mathbf{B}}+\mathbf{J}_{\mathbf{C}}$ and $\mathbf{J}_{\mathbf{A}}+\mathbf{J}_{\mathbf{P}}=\mathbf{J}_{\mathbf{B}}+\mathbf{J}_{\mathbf{C}}+\mathbf{J}_{\mathbf{L}}$. The transition in terms of partial wave amplitudes is

$$
\Gamma=\frac{\pi}{4} \frac{|\vec{P}|}{M_{A}^{2}} \sum_{J L}\left|\mathcal{M}^{J L}\right|^{2}
$$

where $P=|\vec{P}|=\frac{\sqrt{\left[M_{A}^{2}-\left(M_{B}+M_{C}\right)^{2}\right]\left[M_{A}^{2}-\left(M_{B}-M_{C}\right)^{2}\right]}}{2 M_{A}}, M_{A}, M_{B}$, and $M_{C}$ are the masses of the meson $A, B$, and $C$.

\subsection{Mixed states}

Heavy-light mesons are not charge conjugation eigenstates and so mixing can occur among states with the same $J^{P}$ that are forbidden for neutral states [46]. These occur between states with $J=L$ and $S=1$ or 0 [46, 47]. When $J=L=1$, the corresponding mixture angle is $\theta=-54.7^{\circ}$ or $\theta=35.3^{\circ}$ [46, 47]. The two $1^{+}$charmed mesons are the mixtures of the ${ }^{3} P_{1}$ and ${ }^{1} P_{1}$ states:

$$
\left(\begin{array}{c}
\left|\frac{1}{2}, 1^{+}\right\rangle \\
\left|\frac{3}{2}, 1^{+}\right\rangle
\end{array}\right)=\left(\begin{array}{cc}
\cos \theta & -\sin \theta \\
\sin \theta & \cos \theta
\end{array}\right)\left(\begin{array}{c}
\left|{ }^{3} P_{1}\right\rangle \\
\left|{ }^{1} P_{1}\right\rangle
\end{array}\right)
$$

In our calculation, the final states are related to $D(2420) / D(2430)$ and $D_{s_{1}}(2460) / D_{s_{1}}(2536)$, which are the $1^{+}$states in the $D$ and $D_{s}$ meson families, respectively. $D(2420) / D(2430)$ and $D_{s_{1}}(2460) / D_{s_{1}}(2536)$ are the mixing of the ${ }^{3} P_{1}$ and ${ }^{1} P_{1}$ states, which satisfy the above relation(see Eq.7). Thus the helicity amplitude can also be deduced as follows

$$
\left(\begin{array}{l}
\mathcal{M}_{\left.|A\rangle \rightarrow \frac{1}{2}, 1^{+}\right\rangle C}^{J L} \\
\mathcal{M}_{\left.|A\rangle \rightarrow \frac{3}{2}, 1^{+}\right\rangle C}^{J L}
\end{array}\right)=\left(\begin{array}{cc}
\cos \theta & -\sin \theta \\
\sin \theta & \cos \theta
\end{array}\right)\left(\begin{array}{l}
\mathcal{M}_{\left.|A\rangle \rightarrow{ }^{3} P_{1}\right\rangle C}^{J L} \\
\mathcal{M}_{\left.|A\rangle \rightarrow P^{1} P_{1}\right\rangle C}^{J L}
\end{array}\right)
$$

and the decay width can be expressed as

$$
\begin{aligned}
& \left.\Gamma\left(|A\rangle \rightarrow \frac{1}{2}, 1^{+}\right\rangle C\right)=\sum_{J L}\left|\cos \theta \mathcal{M}_{\left.|A\rangle \rightarrow{ }^{3} P_{1}\right\rangle C}^{J L}-\sin \theta \mathcal{M}_{\left.|A\rangle \rightarrow{ }^{1} P_{1}\right\rangle C}^{J L}\right|^{2} \\
& \left.\Gamma\left(|A\rangle \rightarrow \frac{3}{2}, 1^{+}\right\rangle C\right)=\sum_{J L}\left|\sin \theta \mathcal{M}_{\left.|A\rangle \rightarrow{ }^{3} P_{1}\right\rangle C}^{J L}+\cos \theta \mathcal{M}_{\left.|A\rangle \rightarrow{ }^{1} P_{1}\right\rangle C}^{J L}\right|^{2}
\end{aligned}
$$

\section{Numerical Results}


The input parameters in the ${ }^{3} P_{0}$ model mainly include the light quark pair $(q \bar{q})$ creation strength $\gamma$, the SHO wave function scale parameter $R$, and the masses of the mesons and the constituent quarks. The adopted masses of the mesons are listed in TABLE I, and $m_{u}=m_{d}=0.22 \mathrm{GeV}, m_{s}=0.419$ $\mathrm{GeV}$ and $m_{c}=1.65 \mathrm{GeV}$ [48].

TABLE I: The adopted masses of the mesons used in our calculation.

\begin{tabular}{ccccccccc}
\hline \hline States & $M_{\pi^{+}}$ & $M_{\pi^{0}}$ & $M_{K^{+}}$ & $M_{K^{*}}$ & $M_{\eta}$ & $M_{\eta^{\prime}}$ & $M_{D^{+}}$ & $M_{D^{0}}$ \\
\hline Mass $(\mathrm{MeV})$ & 139.57 & 134.9766 & 493.677 & 891.66 & 547.853 & 957.78 & 1869.6 & 1864.83 \\
\hline States & $M_{D_{s}^{*+}}$ & $M_{D_{s}^{+}}$ & $M_{D_{0}^{*}(2400)}$ & $M_{D(2430)}$ & $M_{D_{(2420)}}$ & $M_{D_{s_{0}}^{* \pm}(2317)}$ & $M_{\rho}$ & $M_{\omega}$ \\
\hline Mass$(\mathrm{MeV})$ & 2112.3 & 1968.47 & 2318 & 2427 & 2421.3 & 2317.8 & 770 & 782 \\
\hline States & $M_{D^{*+}}$ & $M_{D^{* 0}}$ & $M_{D_{2}^{*}(2460)}$ & $M_{D_{s_{1}}(2460)}$ & $M_{D_{s_{1}}(2536)}$ & & & \\
\hline Mass $(\mathrm{MeV})$ & 2010.25 & 2006.96 & 2464.4 & 2459.5 & 2535.11 & & & \\
\hline \hline
\end{tabular}

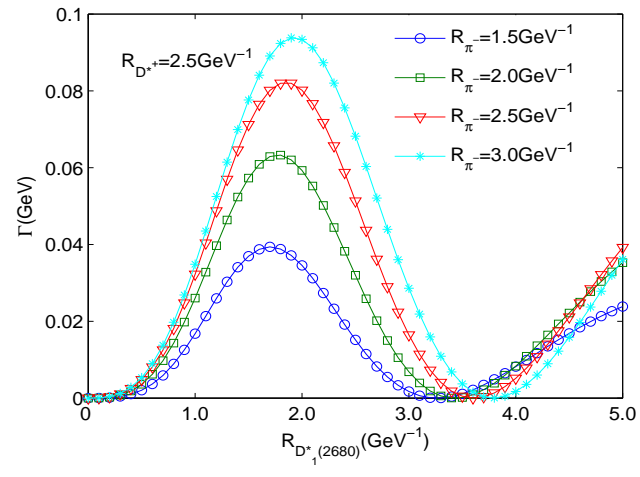

FIG. 2: The strong decay of $D_{1}^{*}(2680) \rightarrow D^{*+} \pi^{-}$ with $R_{D^{*+}}=2.5 \mathrm{GeV}^{-1}$.

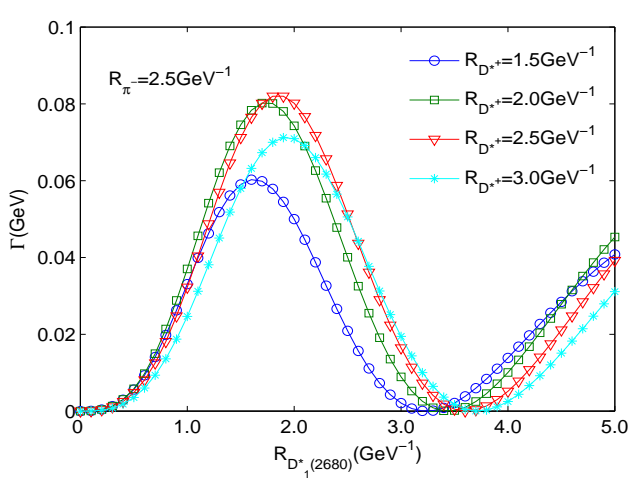

FIG. 3: The strong decay of $D_{1}^{*}(2680) \rightarrow D^{*+} \pi^{-}$ with $R_{\pi^{-}}=2.5 \mathrm{GeV}^{-1}$.

The scale parameter $R$ has a significant influence on the shapes of the radial wave functions. The spatial integral in Eq.3 is sensitive to the parameter $R$, therefore the decay width based on the ${ }^{3} P_{0}$ model is sensitive to the parameter $R$. Taking the decay $D_{1}^{*}(2680) \rightarrow D^{*+} \pi^{-}$as an example, we plot the decay width versus the input parameter $R$ in Figs. 2 and 3. From these two figures, we can easily see the dependence of the decay width on the input parameter $R$. If $R_{D^{*+}}$ and $R_{\pi^{-}}$are all fixed to be $2.5 \mathrm{GeV}^{-1}$ (the lines with triangles in Figs. 2 and 3$)$, the decay width of the $D_{1}^{*}(2680)$ changes several times with the value of $R_{D_{1}^{*}(2680)}$ from $1.5 \mathrm{GeV}^{-1}$ to $3.0 \mathrm{GeV}^{-1}$. Similarly, the decay width changes $2-3$ times, when $R_{D_{1}^{*}(2680)}$ and $R_{\pi^{-}}$(or $R_{D_{1}^{*}(2680)}$ and $R_{D^{*+}}$ ) are fixed to be $2.5 \mathrm{GeV}^{-1}$ while the value of $R_{D^{*+}}\left(\right.$ or $R_{\pi^{-}}$) changes.

Once the optimal values of $\gamma$ and $R$ are determined, the best predictions based on ${ }^{3} P_{0}$ decay model are expected. In reference [20] H.G.Blundel et al. carried out a series of least squares fits of the model predictions to the decay widths of 28 of the best known meson decays, and the common oscillator 
parameter $R$ with a value of $2.5 \mathrm{GeV}^{-1}$ is suggested to be the optimal value. As for the factor $\gamma$, it was also fitted at the same time according to experimental data, giving a fitted value of 6.25 [20]. More detailed analysis of the input parameters in the ${ }^{3} P_{0}$ model can be found in Ref. [20]. Thus, we adopt the SHO wave function with common $R$ whose value is chosen to be $2.5 \mathrm{GeV}^{-1}$. Correspondingly, the $\gamma$ value is chosen to be 6.25 for the creation of $u / d$ quark [20]. As for the strange quark pair $(s \bar{s})$, its creation strength can be related by $\gamma_{s \bar{s}}=\gamma / \sqrt{3}[16]$. As a simple test, we also calculate the decay ratio

TABLE II: The experimental values and numerical result based on the ${ }^{3} P_{0}$ decay model of the ratio $\Gamma\left(D_{2}^{*}(2460) \rightarrow D^{+} \pi^{-}\right)$ $\frac{\Gamma\left(D_{2}^{*}(2460) \rightarrow D^{*+} \pi^{-}\right)}{\Gamma}$

\begin{tabular}{cccccc}
\hline \hline BaBar [49] & CLEO [50] & CLEO [ [51] & ARGUS [52] & ZEUS [53] & $3 \mathrm{P}_{0}$ \\
\hline $1.47 \pm 0.03 \pm 0.16$ & $2.2 \pm 0.7 \pm 0.6$ & $2.3 \pm 0.8$ & $3.0 \pm 1.1 \pm 1.5$ & $2.8 \pm 0.8_{-0.6}^{+0.5}$ & 2.29 \\
\hline \hline
\end{tabular}

$\frac{\Gamma\left(D_{2}^{*}(2460) \rightarrow D^{+} \pi^{-}\right)}{\Gamma\left(D_{2}^{*}(2460) \rightarrow D^{*+} \pi^{-}\right)}$of $D_{2}^{*}(2460)$ meson with the above parameters. The corresponding experimental data from the BaBar [49], CLEO [50, 51], ARGUS [52], and ZEUS [53] collaborations are listed in TABLE II. The present calculation 2.29 based on the ${ }^{3} P_{0}$ model is in agreement well with the average experimental value 2.35. Certainly, we can also predict the decay ratio $\frac{\Gamma\left(D_{2}^{*}(2460) \rightarrow D^{+} \pi^{-}\right)}{\Gamma\left(D_{2}^{*}(2460) \rightarrow D^{*+} \pi^{-}\right)}$with some other methods such as the heavy-quark symmetry theory [54] and the heavy meson effective theory [55]. With the assumption that the transition is dominated by $\bar{u} \rightarrow \pi^{-} d$, the heavy-quark symmetry theory gave the expression of the decay ratio $r=\frac{2}{3}\left(\frac{p}{p^{*}}\right)^{5}=2.44$, where $p=507 \mathrm{MeV}$ and $p^{*}=391 \mathrm{MeV}$ are the c.m. 3-momenta in the decays $D_{2}^{* 0} \rightarrow D^{+} \pi^{-}$and $D_{2}^{* 0} \rightarrow D^{*+} \pi^{-}$, respectively. In reference [55], the heavy meson effective theory almost gave the same expression as it of the heavy-quark symmetry theory. Thus, our calculation is just a primary verification, which indicates that the ${ }^{3} P_{0}$ model with the above parameters can reproduce the experimental data to some extent.

The numerical values of the decay widths and ratios of the charmed mesons $D_{1}^{*}(2680), D_{3}^{*}(2760)$ and $D_{2}^{*}(3000)$ observed by the LHCb collaboration are presented in TABLE III-IV. It can be seen from TABLE III that the total width of $D_{3}^{*}(2760)$ is consistent well with the experimental data of LHCb collaboration, which indicates $D_{3}^{*}(2760)$ is most probably the $1 D \frac{5}{2} 3^{-}$meson. Besides the decay channel $D^{+} \pi^{-}$, the decay ratios in TABLE IV indicates that the other probable decay channels include $D^{*+} \pi^{-}, D^{* 0} \pi^{0}, D_{S}^{+} K^{-}, D^{* 0} \eta, D^{0} \eta$ and $D^{+} \rho$. As for $D_{1}^{*}(2680)$, the total width is predicted to be 208.91MeV which is about $21 \mathrm{MeV}$ above the central value of the experimental data. Considering the total uncertainties of the experimental data, our result is also in agreement with it, which suggests that $D_{1}^{*}(2680)$ can be assigned as the $2 S \frac{1}{2} 1^{-}$state. Besides $D^{+} \pi^{-}, D^{*+} \pi^{-}, D^{0} \pi^{0}, D^{* 0} \eta$ and $D^{* 0} \pi^{0}$ are also its dominant decay channels.

Experiments indicate $D_{2}^{*}(3000)$ is a $2^{+}$state charmed meson [1]. Thus, we study its decay behavior with the $1 F \frac{5}{2} 2^{+}, 2 P \frac{3}{2} 2^{+}, 2 F \frac{5}{2} 2^{+}$and $3 P \frac{3}{2} 2^{+}$assignments. As the candidate of $D_{2}^{*}(3000)$, the total width of $2 F \frac{5}{2} 2^{+}$is predicted to be only $32.09 \mathrm{MeV}$ which is about $150 \mathrm{MeV}$ smaller than the central 
TABLE III: The strong decay widths of $D_{1}^{*}(2680), D_{3}^{*}(2760)$ and $D_{2}^{*}(3000)$ with possible assignments. If the corresponding decay channel is forbidden,we mark it by "-". All values in units of $\mathrm{MeV}$.

\begin{tabular}{|c|c|c|c|c|c|c|}
\hline & \multirow{2}{*}{$\frac{D_{1}^{*}(2680)}{2 S \frac{1}{2} 1^{-}}$} & \multirow{2}{*}{$\frac{D_{3}^{*}(2760)}{1 D \frac{5}{2} 3^{-}}$} & \multicolumn{4}{|c|}{$D_{2}^{*}(3000)$} \\
\hline & & & $1 F \frac{5}{2} 2^{+}$ & $2 P \frac{3}{2} 2^{+}$ & $2 F \frac{5}{2} 2^{+}$ & $3 P \frac{3}{2} 2^{+}$ \\
\hline$D^{*+} \pi^{-}$ & 50.92 & 17.24 & 9.67 & 0.97 & 1.45 & 3.02 \\
\hline$D_{S}^{*+} K^{-}$ & 12.68 & 0.38 & 7.97 & 24.21 & 0.53 & 1.37 \\
\hline$D^{* 0} \pi^{0}$ & 25.53 & 8.85 & 4.76 & 0.43 & 0.75 & 1.55 \\
\hline$D^{* 0} \eta$ & 20.01 & 13.86 & 8.05 & 5.52 & 0.06 & 0.18 \\
\hline$D^{* 0} \eta^{\prime}$ & - & - & 7.75 & 16.58 & 0.87 & 2.10 \\
\hline$D^{+} \pi^{-}$ & 18.17 & 27.51 & 7.17 & 1.11 & 4.85 & 3.86 \\
\hline$D_{S}^{+} K^{-}$ & 22.68 & 2.52 & 10.35 & 11.17 & 0.08 & 0.09 \\
\hline$D^{0} \pi^{0}$ & 8.86 & 14.10 & 3.46 & 0.63 & 2.47 & 1.96 \\
\hline$D^{0} \eta$ & 16.37 & 5.13 & 7.88 & 0.37 & 1.13 & 1.04 \\
\hline$D^{0} \eta^{\prime}$ & - & - & 15.82 & 9.84 & 0.46 & 0.33 \\
\hline$D^{*+} \rho$ & - & - & 15.70 & 100.10 & 0.41 & 7.23 \\
\hline$D_{S}^{*+} K^{*}$ & - & - & 3.27 & 34.87 & 1.09 & 5.74 \\
\hline$D^{* 0} \rho$ & - & - & 7.85 & 50.10 & 0.19 & 3.51 \\
\hline$D^{* 0} \omega$ & - & - & 7.87 & 50.11 & 0.23 & 3.82 \\
\hline$D^{+} \rho$ & 15.97 & 1.18 & 17.44 & 12.51 & 0.09 & 0.28 \\
\hline$D_{S}^{+} K^{*}$ & - & - & 8.01 & 28.72 & 1.31 & 3.39 \\
\hline$D^{0} \rho$ & 9.22 & 0.66 & 8.63 & 6.00 & 0.06 & 0.17 \\
\hline$D^{0} \omega$ & 6.28 & 0.51 & 8.82 & 6.49 & 0.04 & 0.12 \\
\hline$D(2420) \pi^{0}$ & 2.21 & 0.01 & 5.88 & 5.13 & 0.22 & 0.02 \\
\hline$D(2420) \eta$ & - & - & 9.31 & 1.49 & 0.58 & 0.50 \\
\hline$D(2430) \pi^{0}$ & 0.01 & 0 & 0.82 & 0.79 & 0.01 & 1.28 \\
\hline$D(2430) \eta$ & - & - & 1.49 & 1.99 & 1.32 & 0.69 \\
\hline$D_{0}^{*}(2400) \pi^{0}$ & - & - & 0 & 0 & 0 & 0 \\
\hline$D_{0}^{*}(2400) \eta$ & - & - & 0 & 0 & 0 & 0 \\
\hline$D_{S}(2460) K^{-}$ & - & - & 1.61 & 3.45 & 0.52 & 0.34 \\
\hline$D_{S}(2536) K^{-}$ & - & - & 10.14 & 1.39 & 2.78 & 1.47 \\
\hline$D_{2}^{*+}(2460) \pi^{-}$ & - & 0.65 & 16.73 & 39.69 & 5.93 & 10.46 \\
\hline$D_{2}^{* 0}(2460) \pi^{0}$ & - & 0.32 & 8.38 & 19.88 & 2.97 & 5.24 \\
\hline$D_{2}^{* 0}(2460) \eta$ & - & 4.49 & 5.22 & 11.82 & 1.69 & 2.81 \\
\hline$D_{s_{0}}^{*+}(2317) K^{-}$ & - & - & 0 & 0 & 0 & 0 \\
\hline Total width & 208.91 & 97.41 & 220.05 & 442.36 & 32.09 & 62.57 \\
\hline
\end{tabular}


value of the experimental data. Thus, it can be completely excluded from the probable assignments. In addition, it can be seen from TABLE III that the width of $2 P \frac{3}{2} 2^{+}$is about $120 \mathrm{MeV}$ above the upper limit of the experimental data. Thus, $D_{2}^{*}(3000)$ is also impossible to be the $2 P \frac{3}{2} 2^{+}$state. In addition, if $D_{2}^{*}(3000)$ is $3 P \frac{3}{2} 2^{+}$state, its predicted cross section is $62.57 \mathrm{MeV}$ which is smaller about $123 \mathrm{MeV}$ than the central value of the experimental data. Although the calculated total width is just above the lower limit of the experimental data, its branching ratio of the $D^{+} \pi^{-}$decay channel is very small. Thus, $3 P \frac{3}{2} 2^{+}$state is also less likely to be the assignment of $D_{2}^{*}(3000)$.

Although the predicted value of the total width of $1 F \frac{5}{2} 2^{+}$is somewhat bigger than the central value of experimental data, it is within the error range. This indicates $1 F \frac{5}{2} 2^{+}$is most likely to be the assignment of $D_{2}^{*}(3000)$. However, this determination needs to be further verified according to experiments in the future. We can see from Table IV that no decay channel show an obvious advantage over another, while the $D_{2}^{*}(3000)$ resonance is observed by the LHCb Collaboration in the $D^{+} \pi^{-}$channel. One possible explanation about this behavior is that the production cross section of $D_{2}^{*}(3000)$ is so large that the fairly small branching ratio is still observable. If the decay ratios of different decay channels are measured in experiments in the future, this determination can be exactly verified. At present, we can temporarily assign $D_{2}^{*}(3000)$ charmed meson as the $1 F \frac{5}{2} 2^{+}$state, while $2 F \frac{5}{2} 2^{+}, 2 P \frac{3}{2} 2^{+}$and $3 P \frac{3}{2} 2^{+}$states can be excluded temporarily. Nevertheless, these decay predictions for the $2 F \frac{5}{2} 2^{+}, 2 P \frac{3}{2} 2^{+}$and $3 P \frac{3}{2} 2^{+}$states are valuable in further searches for the partners of $D_{2}^{*}(3000)$. For $2 P \frac{3}{2} 2^{+}$as an example, its decay ratios of $D^{*+} \rho, D^{* 0} \rho$ and $D^{* 0} \omega$ is much more obvious than the other decay modes, which can be used as a valuable judgement of this meson.

In reference [12], the decay behavior of $D_{2}^{*}(3000)$ was also analyzed using the ${ }^{3} P_{0}$ decay model. The $3 P \frac{3}{2} 2^{+}$state was predicted as the most possible assignment of the $D_{2}^{*}(3000)$ in their work, while the assignment of the $2 F \frac{5}{2} 2^{+}$charmed meson could not be fully excluded. The primary difference between our analysis and theirs in reference [12] about the $D_{2}^{*}(3000)$ charmed meson is that they employed the SHO wave function with the effective scale parameter $R$ [12], while we adopt the common valve of the scale parameter $R$ which was calculated by fitting the experimental data in reference [20]. Thus, the difference between the results in reference [12] and ours is mainly due to the influence of the input parameter $R$, which needs further confirmation by future experimental data from $\mathrm{LHCb}$ and forthcoming Belle II.

\section{Conclusion}

In this article, we carry out an analysis of the newly observed charmed mesons $D_{1}^{*}(2680), D_{3}^{*}(2760)$ and $D_{2}^{*}(3000)$ reported by $\mathrm{LHCb}$ collaboration with the ${ }^{3} P_{0}$ decay model. Our analysis supports $D_{1}^{*}(2680)$ and $D_{3}^{*}(2760)$ to be the $2 S \frac{1}{2} 1^{-}$and $1 D \frac{5}{2} 3^{+}$assignments separately. In addition, the partial width and ratios are obtained, further shedding light on the nature of these two mesons. The total width predicted by the ${ }^{3} P_{0}$ decay model supports the $1 F \frac{5}{2} 2^{+}$for the $D_{2}^{*}(3000)$ meson, which needs 
further confirmation from the measured partial decay ratios. When investigating $D_{2}^{*}(3000)$, we have also analyzed the decay behavior of the $2 P \frac{3}{2} 2^{+}, 2 F \frac{5}{2} 2^{+}$and $2 P \frac{3}{2} 2^{+}$states, which can be used as valuable judgements for the assignments of the newly observed charmed mesons in the future.

\section{Acknowledgment}

This work is supported by National Natural Science Foundation of China, Grant Numbers 11375063 and the Fundamental Research Funds for the Central Universities, Grant Number 2016MS133 and 13QN59.

[1] R.Aaij et al, arXiv:1608.01289,

[2] P. del Amo Sanchez et al. [BaBar Collaboration], Phys. Rev. D 82 (2010) 111101 arXiv:1009.2076 [hepex]].

[3] R. Aaij et al. [LHCb Collaboration], JHEP 1309 (2013) 145 arXiv:1307.4556.

[4] B. Aubert et al. [BaBar Collaboration], Phys. Rev. D 79 (2009) 112004 arXiv:0901.1291 [hep-ex]].

[5] J. M. Link et al. [FOCUS Collaboration], Phys. Lett. B 586 (2004) 11 hep-ex/0312060.

[6] K. Abe et al. [Belle Collaboration], Phys. Rev. D 69 (2004) 112002 hep-ex/0307021.

[7] B. Aubert et al. [BaBar Collaboration], Phys. Rev. D 74 (2006) 012001 hep-ex/0604009.

[8] J. C. Anjos et al. [Tagged Photon Spectrometer Collaboration], Phys. Rev. Lett. 62 (1989) 1717.

[9] H. Albrecht et al. [ARGUS Collaboration], Phys. Rev. Lett. 56 (1986) 549

[10] K. A. Olive et al, Chin. Phys. C38 (2014) 090001.

[11] Z. G. Wang, arXiv:1608.02176.

[12] J.Z. Wang, D.Y. Chen, Q.T.Song, X Liu, T. Matsuki, arXiv:1608.04186.

[13] L.Micu, Nucl. Phys. B 10, 521(1969).

[14] R. Carlitz and M. Kislinger, Phys. Rev. D 2, 336 (1970); E. W. Colglazier and J. L. Rosner, Nucl. Phys. B27, 349 (1971);W. P. Petersen and J. L. Rosner, Phys. Rev. D 6, 820 (1972).

[15] A.Le Yaouanc, L. Oliver, O. Pene, J-C. Raynal, Phys. Rev. D 8, 2223 (1973); Phys. Rev. D 9, 1415 (1974); Phys. Rev. D 11, 1272 (1975); Phys. Lett. B 71, 397 (1977).

[16] A. Le Yaouanc, L. Oliver, O. Pene, and J. C. Raynal, Phys. Lett. B 72, 57 (1977).

[17] A. Le Yaouanc, L. Oliver, O. Pene, J-C. Raynal, Hadron transitons in the quark model(Gordon and Breach Science Publishers, New York, 1988)

[18] W. Roberts and B. Silvestr-Brac, Few-Body Syst. 11, 171 (1992)

[19] S.Capstick, N. Isgur, Phys. Rev. D 34, 2809 (1986); S. Capstick, W. Roberts, Phys. Rev. D 494570 (1994).

[20] H. G. Blundell, hep-ph/9608473 H.G. Blundell, S. Godfrey, Phys. Rev. D 53,3700 (1996); H. G. Blundell, S. Godfrey, B. Phelps, Phys. Rev. D 53, 3712 (1996).

[21] H. Q. Zhou, R. G. Ping, B. S. Zou, Phys. Lett. B 611, 123 (2005); G. J. Ding, M. L. Yan, Phys. Lett. B 657, 49 (2007).

[22] Bing Chen, Deng-Xia Wang and Ailin Zhang, Phys.Rev. D 80, 071502(2009). 
[23] De-Min Li, Shan Zhou, Phys.Rev.D 78, 054013 (2008); De-Min Li, En Wang, Eur.Phys.J.C 63:297 (2009); De-Min Li, Peng-Fei Ji, Bing Ma, Eur.Phys.J.C 71,1582 (2011)

[24] B. Zhang, X. Liu, W. Z. Deng, S. L. Zhu, Eur. Phys. J. C 50, 617 (2007); Y. Sun, Q. T. Song, D.Y. Chen, X. Liu and S. L. Zhu, arXiv:1401.1595 [hep-ph](2013).

[25] Y. C. Yang, Z. R. Xia, J. L. Ping, Phys.Rev.D 81,094003(2010).

[26] E. S. Ackleh, T. Barnes and E. S. Swanson, Phys. Rev. D 54, 6811 (1996); T. Barnes, N. Black and P. R. Page, Phys. Rev. D 68, 054014 (2003); T. Barnes, S. Godfrey and E. S. Swanson, Phys. Rev. D 72 , 054026 (2005)

[27] J. Ferretti, G. Galat'a and E. Santopinto, Phys. Rev. C 88, 015207 (2013).

[28] J. Ferretti, G. Galat'a and E. Santopinto, Phys. Rev. D 90, 054010 (2014).

[29] J. Ferretti and E. Santopinto, Phys. Rev. D 90, 094022 (2014).

[30] F. E. Close, E. S. Swanson, Phys. Rev. D 72, 094004 (2005); F. E. Close, C. E. Thomas, O. Lakhina, E. S. Swanson, Phys. Lett. B 647, 159(2007);

[31] J. Segovia, D. R. Entem and F. Fernandez, Phys. Lett. B 715, 322 (2012).

[32] P. del Amo Sanchez et al, Phys. Rev. D82 (2010) 111101.

[33] R. Aaij et al, JHEP 1309 (2013) 145.

[34] Z. G. Wang, Phys. Rev. D83 (2011) 014009.

[35] P. Colangelo, F. De Fazio and S. Nicotri, Phys. Lett. B642 (2006) 48; P. Colangelo, F. De Fazio, F. Giannuzzi and S. Nicotri, Phys. Rev. D86 (2012) 054024.

[36] A. M. Badalian and B. L. G. Bakker, Phys. Rev. D84 (2011) 034006; Q. F. Lu and D. M. Li, Phys. Rev. D90 (2014) 054024; Q. T. Song, D. Y. Chen, X. Liu and T. Matsuki, Phys. Rev. D92 (2015) 074011; B. Chen, X. Liu and A. Zhang, Phys. Rev. D92 (2015) 034005.

[37] Z. G. Wang, Phys. Rev. D88 (2013) 114003.

[38] S. Godfrey and K. Moats, Phys. Rev. D93 (2016) 034035.

[39] R. Aaij et al, Phys. Rev. D92 (2015) 032002.

[40] X. H. Zhong, Phys. Rev. D82 (2010) 114014; D. M. Li, P. F. Ji and B. Ma, Eur. Phys. J. C71 (2011) 1582.

[41] S. Godfrey and N. Isgur, Phys. Rev. D 32, 189(1985).

[42] M. Di Pierro and E. Eichten, Phys. Rev. D 64, 114004(2001).

[43] D. Ebert, R. N. Faustov and V. O. Galkin, Eur. Phys. J. C 66,197(2010).

[44] C. Hayne and N. Isgur, Phys. Rev. D 25, 1944 (1982)

[45] M. Jacob, G. C. Wick, Ann. Phys. 7, 404 (1959)

[46] A.De Rujula, H.Georgi, and S.L.Glashow,Phys. Rev. Lett. 37, 398, 785 (1977)

[47] T. Matsuki, T. Morii,K. Seo, Prog.Theor.Phys. 124,285(2010)

[48] K. A. Olive et al. [Particle Data Group Collaboration], Chin. Phys. C 38, 090001 (2014). doi:10.1088/16741137/38/9/090001

[49] P.del Amo Sanchez et al, Phys.Rev. D 82,111101(2010).

[50] P. Avery et al, Phys. Lett. B 331, 236(1994).

[51] P. Avery et al, Phys. Rev. D 41, 774(1990).

[52] H. Albrecht et al, Phys. Lett. B 232, 398(1989). 
[53] S. Chekanov et al, Eur. Phys. J. C 60, 25(2009).

[54] J. L. Rosner, Comments on Nucl. and Part. Phys. 16, 109 (1986); N. Isgur and M. Wise, Phys. Rev. Lett. 66, 1130 (1991); Ming-Lu, Mark B. Wise, and Nathan Isgur, Phys. Rev. D 45, 1553 (1992).

[55] Z.G. Wang, Phys. Rev.D 88, 114003 (2013). 
TABLE IV: The decay ratios of partial decay width $\Gamma_{p} / \Gamma_{T}$ of $D_{1}^{*}(2680), D_{3}^{*}(2760)$ and $D_{2}^{*}(3000)$ with possible assignments.

\begin{tabular}{|c|c|c|c|c|c|c|}
\hline & \multirow{2}{*}{$\frac{D_{1}^{*}(2680)}{2 S \frac{1}{2} 1^{-}}$} & \multirow{2}{*}{$\frac{D_{3}^{*}(2760)}{1 D \frac{5}{2} 3^{-}}$} & \multicolumn{4}{|c|}{$D_{2}^{*}(3000)$} \\
\hline & & & $1 F \frac{5}{2} 2^{+}$ & $2 P \frac{3}{2} 2^{+}$ & $2 F \frac{5}{2} 2^{+}$ & $3 P \frac{3}{2} 2^{+}$ \\
\hline$D^{*+} \pi^{-}$ & 0.24 & 0.18 & 0.04 & 0.002 & 0.05 & 0.05 \\
\hline$D_{S}^{*+} K^{-}$ & 0.06 & 0.004 & 0.04 & 0.05 & 0.02 & 0.02 \\
\hline$D^{* 0} \pi^{0}$ & 0.12 & 0.09 & 0.02 & 0.001 & 0.02 & 0.02 \\
\hline$D^{* 0} \eta$ & 0.10 & 0.14 & 0.04 & 0.01 & 0.002 & 0.003 \\
\hline$D^{* 0} \eta^{\prime}$ & - & - & 0.04 & 0.04 & 0.03 & 0.03 \\
\hline$D^{+} \pi^{-}$ & 0.09 & 0.28 & 0.03 & 0.003 & 0.15 & 0.06 \\
\hline$D_{S}^{+} K^{-}$ & 0.11 & 0.03 & 0.05 & 0.03 & 0.003 & 0.001 \\
\hline$D^{0} \pi^{0}$ & 0.04 & 0.14 & 0.02 & 0.001 & 0.08 & 0.03 \\
\hline$D^{0} \eta$ & 0.08 & 0.05 & 0.04 & 0.0008 & 0.04 & 0.02 \\
\hline$D^{0} \eta^{\prime}$ & - & - & 0.07 & 0.02 & 0.01 & 0.005 \\
\hline$D^{*+} \rho$ & - & - & 0.07 & 0.23 & 0.01 & 0.12 \\
\hline$D_{S}^{*+} K^{*}$ & - & - & 0.01 & 0.08 & 0.03 & 0.09 \\
\hline$D^{* 0} \rho$ & - & - & 0.04 & 0.11 & 0.006 & 0.06 \\
\hline$D^{* 0} \omega$ & - & - & 0.04 & 0.11 & 0.007 & 0.06 \\
\hline$D^{+} \rho$ & 0.08 & 0.01 & 0.08 & 0.03 & 0.003 & 0.004 \\
\hline$D_{S}^{+} K^{*}$ & - & - & 0.04 & 0.06 & 0.04 & 0.05 \\
\hline$D^{0} \rho$ & 0.04 & 0.007 & 0.04 & 0.01 & 0.002 & 0.003 \\
\hline$D^{0} \omega$ & 0.03 & 0.005 & 0.04 & 0.01 & 0.001 & 0.002 \\
\hline$D(2420) \pi^{0}$ & 0.01 & 0.0001 & 0.03 & 0.01 & 0.007 & 0.0003 \\
\hline$D(2420) \eta$ & - & - & 0.04 & 0.003 & 0.02 & 0.008 \\
\hline$D(2430) \pi^{0}$ & 0 & 0 & 0.004 & 0.002 & 0.0003 & 0.02 \\
\hline$D(2430) \eta$ & - & - & 0.007 & 0.005 & 0.04 & 0.01 \\
\hline$D_{0}^{*}(2400) \pi^{0}$ & - & - & 0 & 0 & 0 & 0 \\
\hline$D_{0}^{*}(2400) \eta$ & - & - & 0 & 0 & 0 & 0 \\
\hline$D_{S}(2460) K^{-}$ & - & - & 0.007 & 0.008 & 0.02 & 0.005 \\
\hline$D_{S}(2536) K^{-}$ & - & - & 0.05 & 0.003 & 0.09 & 0.02 \\
\hline$D_{2}^{*+}(2460) \pi^{-}$ & - & 0.007 & 0.08 & 0.08 & 0.18 & 0.17 \\
\hline$D_{2}^{* 0}(2460) \pi^{0}$ & - & 0.003 & 0.04 & 0.04 & 0.09 & 0.08 \\
\hline$D_{2}^{* 0}(2460) \eta$ & - & 0.05 & 0.02 & 0.03 & 0.05 & 0.04 \\
\hline$D_{s_{0}}^{*+}(2317) K^{-}$ & - & - & 0 & 0 & 0 & 0 \\
\hline
\end{tabular}

